\title{
An Integrated Model of Sustainability and Emission Control: The Concept of Society as a Super Organism That Lives by Consuming Its Own Waste Using Alternative Energy as Currency
}

\author{
Paul A. Comet \\ Comet Environmental Consulting, Houston, Texas, USA \\ Email address: \\ pcomet@prodigy.net
}

\section{To cite this article:}

Pail A. Comet. An Integrated Model of Sustainability and Emission Control: The Concept of Society as a Super Organism That Lives by Consuming Its Own Waste Using Alternative Energy as Currency. American Journal of Environmental Protection. Special Issue: New Technologies and Geoengineering Approaches for Climate. Vol. 5, No. 3-1, 2016, pp. 17-20. doi: 10.11648/j.ajep.s.2016050301.13

\begin{abstract}
There is a need for new political, social and economic theories, as neither present day capitalism nor communism are yet adequate in creating sustainable societies, or for reversing climate change. A rough "working" document for discussion purposes.
\end{abstract}

Keywords: Sustainability, Economic Theories, Alternative Energy, Super Organism

\section{Introduction}

"Strategies and actions can be pursued now which will move towards climate-resilient pathways for sustainable development, while at the same time helping to improve livelihoods, social and economic well-being and effective environmental management. In some cases, economic diversification can be an important element of such strategies. The effectiveness of integrated responses can be enhanced by relevant tools, suitable governance structures and adequate institutional and human capacity (medium confidence). Integrated responses are especially relevant to energy planning and implementation; interactions among water, food, energy and biological carbon sequestration; and urban planning, which provides substantial opportunities for enhanced resilience, reduced emissions and more sustainable development (medium confidence)" [1].

\section{Concepts}

A series of simple concepts are combined here for the creation of carbon- neutral population centers with sustainable economic systems.

The primary concept is the observable operation of an "ecosphere"; a small glass container, welded shut containing sea water, a bubble of air, a spring of algae and a few tiny shrimps. A small patch of basal gravel completes, the ensemble. Placed by a window the ecosphere can be indefinitely sustainable. It was developed by NASA. Somehow the shrimps "know" not to eat up all the algae by overbreeding. Perhaps the shrimps secrete an "overcrowding" pheromone.

A second concept is the idea of a "superorganism": this can mean many things from "Gaia" at the planetary level $[2,3]$; to bees, ants and other social organisms; which may (as in the case of ants and bees) have delegated their reproductive rights to a single individual (queen) or retained those rights (prairie dogs \&, humans). At the smallest scale are symbiotic organelles that allow autotrophy within "animal like", otherwise heterotrophic, protists; these marvelous "wee beasties" can also be considered to "micro super organisms" which may or may not be colonial. Various protists come to mind. All of these examples should be indefinitely sustainable given enough water, air $\&$ sunlight.

The third concept is the concept of waste recycling; not just by the reuse or repairing of damaged, unwanted, surplus, or decayed commodities, packaging etc. but also by totally deconstructing materials by combustion (or consumption by detritovores) and using the recoverable energy, ash and gas for other purposes such as heating \&fertilizer, Comet, P.A., 2006, 
2007, 2010. [4-6]. This concept links closely to the ecosphere where everything is indefinitely recycled.

A fourth concept is the idea that money can be denominated in units of energy - joules or watts. Fawzi, M, 2009, [7].

\section{Proposed Implementation}

If each city could be self - reliant, using local crops as food (possibly "twinning" a rural town and larger cities) the city become reliant on urban areas for food and the rural town using effluent, as fertilizers and other recycled materials from the city, then a symbiotic arrangement might emerge. Symbiosis might be facilitated by local electrical grids which covers both rural country side and the large city. If alternative energy forms the basis of a single credit card "banking system", external to the present one, then a series of fully autonomous regions may be created. The waste stream then forms a resource of stored energy, much better than batteries, for night time, electricity or day peak demand. Similarly the incombustibles, such as steel and aluminum cans, could form the basis of local manufacturing. Other waste such as glass and ceramics could be used in decorative construction work such as sculpture. Plastics can be recycled for a multitude of purpose or used as fuel in a gasifier.

The value of superorganism theory is that it may potentially offer a predictive, integrated, holistic approach to understanding how a healthy society functions - economically, politically, sociologically, maybe even spiritually. Medical analogies then may become useful \& the idea of societal ills being curable may become valid, Comet, P.A., 2015, [8]. The question then becomes what is the best biological analogy? Gaia, unicellular organisms, multicellular organisms, plants, animals, \& symbiotic associations, particularly those that combine autotrophy and heterotrophy? Though the present model takes fragments of each of these analogies, it is initially the concept of a single photosynthetic cell, the basic functioning unit of any plant that seems to fit best to city \& state, as the cell defines the basic parameters of self and non-self, chemical factories for creating the compounds of life, derived ultimately from sunshine, in the chloroplasts, partly analogous to "solar" or photovoltaic panels" that can place energy into storage as lipids \& carbohydrates, cellular infrastructure such as walls, water reservoirs, pipelines between cells functioning by osmosis - the list is long. Many land plants (even cyanobacteria), need little but water, air, sunshine. However, nitrogen and phosphorus sources may require bacteria $\&$ fungi to collect these vital elements for the plant, using the plants stored photosynthate as "payment" to the fungus etc. Hence, the "currency" of this system is stored energy ultimately derived from the sun, it does not matter what the organism is. If one perceives that each town or city can be compared to heterotrophic nucleus, and the surrounding fields are photosynthetic chloroplasts; then roads and railways can be compared to veins, power lines and telecommunications to a neural system. However, it is the circulatory fluid (blood etc.) that can be compared to the modern banking system and the flow of money, as well as those resources that money can buy.

Though the above analogies are imperfect, they are useful in the sense that certain features of civilization, presently arcane, such as the novel identification of money as "energy tokens"; the health of a society or individuals, measured in not only in terms of conventional personal medical "wellness", but also in terms of social problems of unemployment, incarceration, substance abuse, domestic violence, divorce, homicide, child abuse, availability of clean water, healthy food, access to good medical care, degree of pollution, waste elimination, longevity etc.; all become group "symptoms" of the health (or lack thereof) of that society. If the group symptoms described above become overwhelming, then political metastasis ensues and the society collapses into war and anarchy. Essentially the "organs of state" can be compared to the organs of a body or the organelles of a single cell. If these malfunction, then sickness, death and disintegration ensues. The Middle East can be used as an example of what can go wrong.

Ultimately if the above models have any validity, then urban and rural areas can be reappraised in terms of a mutually beneficial economic "modular" structure, just using the waste stream and sunshine. By using some of these ideas, the first sustainable lunar base (or autonomous refugee camps, i.e. not requiring external charity, just land \& water) may be viable.

Essentially we are advocating cooperative capitalism in which the municipal structure forms the basic administrative unit, this might be urban or rural; or best of all, both linked together by a common grid based on alternative energy, this system emphasizes the waste stream as stored energy, as well as solar, wind etc. for immediate use. During night time, or peak electricity demand, the energy stored in the organic materials of the waste stream can be accessed by fermentation, gasification, or combustion etc.; also utilizing cogeneration to also access the energy in "waste heat" produced by those processes. The electric grid might form a common bank of the 2 communities, in which personal credit cards monitor energy input and withdrawal from the grid.

If rural food production and urban manufacturing are correctly balanced, just by using the waste stream \& alternative energy, then the population may become autonomous, with potentially higher levels of employment for those "left behind" in the present system. The existing system could be left intact. However the pressure to import raw materials from elsewhere would be much reduced. For instance, using the example of animal bones, a simple model can be proposed. These are the phosphate islands of the Pacific, for not until the last ton of phosphate be removed from these "disappearing" islands, will people routinely start recycling food bones as fertilizer. The concentrated phosphate deposits will always require less energy ( $\&$ money) for extraction \& processing, as compared to food bones which would need to be laboriously collected. Essentially the present system of "cheapest resource to be used first" based on fossil fuels, results in "material entropy" (a better term would be resource dilution or dispersal) and the eventual loss of everything ( $\&$ everybody) to the landfill, cemetery, (dole or 
prison).The "rag \& bone" man of Dickensian times maybe need be reintroduced. If the currency needed to pay the bone collector comes from the local grid, then the system becomes sustainable \& the phosphate is indefinitely retained by the municipality. The money also should circulate indefinitely within the community, as long as the sun shines $\&$ as long as the credit card is not usable in other municipalities.

A difficulty to be overcome are that is that articles made for sale, may trade for considerably more elsewhere (outside the municipal grid),than they cost energy - wise to manufacture, also currency conversion between the existing system \& the energy - based one proposed here, has not yet been solved. This will not be a problem immediately, as there is probably insufficient energy to be yet used in manufacturing. However, as new technology is introduced to better capture, use and store the sun's rays, there will eventually be a surplus of energy that can be used in local manufacturing. Customers from another grid could purchase items, or alternatively, replenish their own grid with more energy from the vending grid etc., that would continually revalidate the energy currency unit.

Once the idea of stored energy is introduced the model becomes much more complex. For an individual to purchase an item, for which the energy to manufacture it has been dispersed, a currency token would become a promissory note similar to those of the present system. Eventually the number of unbacked promissory notes could become overwhelming and the system collapses. However social organisms, plants etc. have solved this problem, for instance a tree that generates dead structural material (trunk) so that the cambium \& leaves etc. can live \& be supported in space. Termite mounds are similar, or virtually any "infrastructure" such as a skeleton, or shell of some kind. As noted above, the replenishment of the grid by new energy would continually validate circulating currency, but stored energy from biochar, batteries, living biomass \& the waste stream might permit savings \& investment.

By analogy with the ecosphere discussed earlier, a specific rate of energy incorporation and loss can be directly observed as one moves through the carbon cycle of photosynthesis, respiration and decomposition. Manufacturing of plant tissue, shrimp tissue and bacterial biomass is regulated by light energy and the continual regeneration of plant material from simple nutrients. Hence by transposing this ecological system to human economic systems, the amount of energy used or absorbed per unit of time should correspond to the number of currency units available. Essentially the cliché "money is power" would be validated.

So when photosynthesis, respiration and decomposition are in balance and confined to a specific area, such as in the ecosphere, the law of conservation of energy might be applied to the printing of "shrimp" currency. However, leaving the existing currency in place \& just using an energy - based credit card for electricity addition / withdrawal, or for articles manufactured, or services provided, by alternative energy, might be easier. The amount of energy in the closed system can be expressed as a limited joule or watt currency as long as it bears a resemblance of amount of both free \& "bound" energy available. The currency problem becomes much more difficult to solve when the system is open and may indeed correspond to "Laissez faire". Conventional capitalism automatically transfers wealth to the least expensive manufacturer or service provider. This problem might be avoided by a number of autonomous areas that are defined by either a gas or electrical grid that is used not only for running domestic appliances, light etc. but eventually in manufacturing \& specifically oriented towards recycling in the waste stream by using it as a manufacturing \& energy feedstock. The credit card used in one autonomous area would, initially, perhaps, not be valid in another area, except under highly controlled circumstances. Eventually manufacturing etc. in one place, that might complement manufacturing of different goods in other places, could justify use a common energy - based credit card.

If it becomes economically feasible to replace fossil with alternative fuels then, and if, this trend were to become widespread, carbon neutrality could be accomplished. The seas \& boreal forests would continue to absorb 2 ppm per year of CO2 (spring "blooms" on land and sea) as indicated from the Mauna Loa observatory "zig - zag" CO2 record of atmospheric concentrations known as the Keeling curve [9]. However this absorption presently removes both $\mathrm{CO} 2$ derived from decomposition of leaf litter $\&$ annual vegetation as well as from fossil fuels. Dead vegetation may have to be charred to fix at least half of this carbon on the land. A range of other techniques for carbon fixation will also be needed to move from carbon neutrality to carbon negativity.

\section{Conclusion}

The observation of the NASA "ecosphere" allows one to mentally model a carbon - neutral, sustainable society, that uses all its waste as a source of stored energy (where city \& state replace the shrimps!) as well as sunlight, wind etc. The creation of an alternative energy - based currency "photonic dollar" is suggested that would gradually outcompete the petrodollar as domestic waste to energy, \& other alternative energy devices are attached to a communal electric grid (that also functions as a bank) that gradually replace fossil fuel energy.

"Superorganism" theory is advocated as a way of distributing resources in an effective manner \& as a way of understanding the complexities of how human society functions in a holistic manner. Societal ills then become diagnosable in terms of a malfunction of the entire social \& economic system. The interposing of a separate economic "tier" specifically for alternative resources \& energy is suggested as a non - revolutionary way of creating sustainable employment [10].

These ideas are proposed because neither the theories of Adam Smith nor Karl Marx address the waste problem, nor clearly identify the "energy nature" of capital. If this new combination of models is successful in solving climate change problems, then economic philosophical differences between competing nation states might prove to be resolvable. 


\section{References}

[1] IPCC Climate Change Synthesis Report Summary for Policymakers, 2014, http://www.ipcc.ch/pdf /assessment-report/ar5/syr/AR5_SYR_FINAL_SPM.pdf

[2] J.E. Lovelock, "Gaia as seen through the atmosphere", Atmospheric Environment, 1972, pp. 579- 580.

[3] J.E. Lovelock, and L. Margulis, Atmospheric and homeostatic by and for the biosphere: the gain hypothesis, Tellus XXVI, 1974, vol 1-2, pp. 1-10.

[4] Comet, P.A “A plan for sustainability”, C\&EN News, vol. 85, issue 13, August 2007, pp.6, 8.

[5] P.A Comet, "Good use of waste". C\&EN News, vol. 84, Numb. 41, October 2006, pp. 4-5,
[6] P.A Comet, "Biochar for CO2 Reduction", C\&EN News, vol. 88, issue11, March 2010, p.4

[7] Fawzi, Marc., 2009, http://p2pfoundation.net/P2P_Energy Economy.

[8] P.A. Comet, "A "lunatic" model of sustainability", 19th Annual Green Chemistry \& Engineering Conference, July 14 to 16th 2015, Bethesda, Maryland, USA.

[9] Latest reading, October 2015. Mauna Loa Observatory, https://scripps.ucsd.edu/programs/keelingcurve/

[10] P.A. Comet, "Planetary regulation of greenhouse gases by changing municipal waste management practice: a commonsense approach. Presentation at American Chemical Society", Green Chemistry Symposium” July 16, 2012. 